\title{
La empresa de base tecnológica y su contribución a la economía mexicana en el periodo 2004-2009
}

\author{
The technology-based firm and its contribution to the Mexican economy \\ in the period 2004-2009
}

\author{
Moisés Alejandro Alarcón Osuna ${ }^{\mathrm{a}, *}$ y Claudia del Carmen Díaz Pérez ${ }^{\mathrm{b}}$ \\ a Universidad Autónoma de Sinaloa, México \\ ${ }^{\mathrm{b}}$ Universidad Autónoma Metropolitana, México
}

Recibido el 30 de julio de 2014; aceptado el 10 de febrero de 2015

Disponible en Internet el 20 de octubre de 2015

\begin{abstract}
Resumen
El propósito de este artículo es analizar la contribución de las empresas de base tecnológica (EBT) a la economía mexicana. El estudio incluye la revisión de los sectores donde participa la EBT, así como la participación que tienen los 4 estratos de empresas por su tamaño. La comparación enfatiza la aportación de las pymes y de la empresa grande en la generación de valor agregado en los sectores en donde se localizan, enfatizando su desempeño general. Los hallazgos principales indican que las EBT mexicanas contribuyen al PIB manufacturero nacional con un poco más del 20\%, a pesar de que constituyen solo el $2 \%$ de las empresas mexicanas. Asimismo, se identificó que son las microempresas las que tienen el más alto valor agregado, pero son las grandes empresas las que tienen una contribución mayor a la economía. Adicionalmente se encontró que los rendimientos crecientes de escala no son una condición que afecte al desempeño de la EBT. Derechos Reservados (C 2015 Universidad Nacional Autónoma de México, Facultad de Contaduría y Administración. Este es un artículo de acceso abierto distribuido bajo los términos de la Licencia Creative Commons CC BY-NC-ND 4.0.
\end{abstract}

Palabras clave: Empresa de base tecnológica; Economía mexicana; Tamaño de empresa; Desempeño de la empresa

\begin{abstract}
The purpose of this article is to analyze the contribution of the technology-based firms (TBF) to the

\footnotetext{
* Autor para correspondencia.

Correo electrónico: alarcon93@ hotmail.com (M.A. Alarcón Osuna).

La revisión por pares es responsabilidad de la Universidad Nacional Autónoma de México.
} Mexican economy. The study includes the revision of the sectors in which the TBF participates as well as the 
participation of the 4 firm strata by their size. The comparison emphasizes the contribution of the SME and the big-sized enterprises on the added value generation in the sectors where they are placed, emphasizing the general performance. The main highlights point that the Mexican TBF contributes on little more than $20 \%$ of the national manufacturing GDP despite being just the $2 \%$ of the total Mexican firms. Also it was identified that the micro-sized firms has the highest added value but the big-sized firms are the ones with major economy contribution. Additionally it was found that the increasing returns of scale are not a condition that affects on the performance of the TBF.

All Rights Reserved @ 2015 Universidad Nacional Autónoma de México, Facultad de Contaduría y Administración. This is an open access item distributed under the Creative Commons CC License BY-NC-ND 4.0.

Keywords: Technology-based firm; Mexican economy; Firm size; Firm performance

\section{Introducción}

El propósito de este artículo es analizar el desempeño de la empresa de base tecnológica (EBT) mexicana a partir de sus aportaciones por estrato según su tamaño a la economía. El estudio de la EBT ha crecido en importancia en las últimas décadas por la relevancia que tienen estas empresas en la generación de mayores niveles de empleo, en el crecimiento en ventas, el valor agregado y su dinámica de innovación que alienta a la economía en su conjunto (Bollinger, Hope y Utterback, 1983; Granstrand, 1998; Autio y Yli-Renko, 1998). La alta productividad de las EBT es uno de los principales argumentos para su estudio (Ortin y Vendrell, 2014; Yagüe y Mach, 2013; Li, Qian y Qian, 2012; Ganotakis y Love, 2011). Asimismo, una de las ventajas más destacadas de este tipo de empresas es su capacidad para generar conocimiento (Granstrand, 1998; Autio y Yli-Renko, 1998; Colombo y Grilli, 2005; Coeurderoy y Murray, 2008; Fong y Alarcón, 2010). El conocimiento producido está distribuido en los dispositivos que establece la empresa, en sus productos y, principalmente, en sus empleados, quienes con relativamente poco capital pueden producir una mayor cantidad de bienes con mayor valor agregado.

Es importante destacar que las pequeñas y medianas empresas (pymes) en general son las principales fuentes de empleo y crecimiento económico (Kulicke y Krupp, 1987); de hecho, las pymes constituyen el $99.5 \%$ de las $\mathrm{EBT}^{1}$. Sin embargo, ha sido difícil la cuantificación de la aportación de este tipo de empresas al total de la economía de cualquier país (Autio y YliRenko, 1998). Algunos autores (Granstrand, 1998, Autio et al., 1998, Wu y Wang, 2007; Ortin y Vendrell, 2014) señalan que la productividad de la EBT no depende de su tamaño, ya que gran parte del valor agregado que estas generan está incluido en el conocimiento, que a su vez está depositado en los productos (Granstrand, 1998, Autio y Yli-Renko, 1998, Wu y Wang, 2007; Ortin y Vendrell, 2014), lo cual les permite trabajar en un estrato de empresas de tamaño pequeño o mediano. Adicionalmente, se ha mostrado evidencia de rendimientos crecientes de escala en la EBT debidos a la diversificación tecnológica (Tsvetkova, Thill y Strumsky, 2014; Li et al., 2012; Patel y Pavitt, 1994; Pavitt, Robson y Townsend, 1989). A pesar de ello no ha sido posible alcanzar un consenso en la definición de sectores económicos en los cuales se agrupen este tipo de empresas, y esta carencia de un consenso ha llevado a caracterizar la EBT con diferentes metodologías.

\footnotetext{
${ }^{1}$ Con la siguiente distribución: el $94 \%$ son pequeñas, el $5.5 \%$, pequeñas, y el $0.5 \%$, grandes, de acuerdo con las estimaciones de Ganotakis y Love (2011).
} 
- De acuerdo con la tecnología que utilizan las empresas. Butchart (1987) y Lall (2000) han definido los sectores tecnológicos como de alto o bajo nivel, basándose en sus gastos de R\&D y el crecimiento económico acelerado en periodos de tiempo relativamente cortos.

- De acuerdo con ejercicios de medición (Breschi, Lenzi, Malerba y Mancusi, 2014; Ejermo y Xiao, 2013; Yagüe y Mach, 2013; Kollmer y Dowling, 2004; Granstrand, 1998; Storey y Tether, 1998a, 1998b; Bollinger et al., 1983), en donde señalan la importancia de otras variables como la edad, la cantidad de empleados, la educación y el género de los administradores y empleados.

- De acuerdo con el entorno inestable y/o turbulento en el cual compiten (Teixeira y Tavares, 2014; Clarysse, Bruneel y Wright, 2011; Suzuki de Oliveira, Teixeira de Freitas, Ferreira y Real, 2011; Aspelund, Berg y Skjevdal, 2005; Kollmer y Dowling, 2004; Granstrand, 1998).

- De acuerdo con el riesgo que implica la diversificación tecnológica sobre las ventas (Kulicke y Krupp, 1987; Fontes y Coombs, 2001; Li et al., 2012; Onetti, Zucchella, Jones y McDougall, 2012).

- De acuerdo con el origen de la empresa. En este aspecto se hace una clara distinción entre el desempeño de una EBT independiente y una spin-off universitaria, donde la última se constituye a partir del conocimiento y el apoyo de una universidad o centro de investigación (Ortin y Vendrell, 2014; Suzuki de Oliveira et al., 2011).

Las clasificaciones anteriores tienen un papel importante en la definición de una EBT; sin embargo, aún no está del todo clara la definición de un sector de base tecnológica, aunque todo ello constituye un precedente de estudios en donde se ha intentado realizar una taxonomía de los sectores de base tecnológica.

En el presente artículo se propone una clasificación de sector de base tecnológica con el cual se obtendrán mediciones sobre la aportación de estos sectores al funcionamiento de la economía nacional. La medición se realiza a partir de los censos económicos de INEGI con base en el SCIAN, del mismo modo que ya ha sido tomado en cuenta en estudios de supervivencia de las EBT (Tsvetkova et al., 2014), pero limitándose solo a la industria del software y electrónica. En particular se pretende describir la aportación de cada uno de los sectores tecnológicos y su evolución en los censos económicos de 2004 y 2009. Se ha seleccionado dicho periodo dado que a partir de los censos económicos de 1999 se cambió el esquema de administración de bases de datos de INEGI en los censos económicos, pasando de la clasificación CMAP a la clasificación SCIAN. Sin embargo, los censos de 1999 se trabajaron con ambos métodos de clasificación (CMAP y SCIAN), perdiendo de esta manera la especificidad de muchas ramas del SCIAN, por lo que los datos de 1999 no resultan del todo comparables con los datos de los últimos censos disponibles (2004 y 2009). Por otra parte, los resultados de los censos de 2014 no se dieron a conocer hasta febrero de 2015, por lo que fue imposible tomarlos en cuenta para este trabajo. Adicionalmente, se propone un modelo de regresión tipo Cobb-Douglas en el cual se estima la productividad factorial del trabajo y el capital. Esta estimación permite analizar en qué medida los sectores de base tecnológica funcionan con economías de escala constantes o crecientes, lo cual mostraría si estos sectores son eficientes en el uso de sus recursos. Este argumento se deriva de la literatura sobre EBT, ya que reconoce que no es necesario para estas empresas lograr una escala de producción masiva para lograr el éxito² (Ortin y Vendrell, 2014; Yagüe y Mach, 2013;

\footnotetext{
2 Éxito se define en la presente investigación como: el sostenimiento de beneficios extraordinarios mediante la obtención de una ventaja competitiva, lo cual ayudará a la empresa en su supervivencia a lo largo del tiempo; en este caso se operacionaliza el éxito como la autosostenibilidad financiera, lo que implica su supervivencia.
} 
Li et al., 2012; Ganotakis y Love, 2011; Wu y Wang, 2007; Granstrand, 1998; Autio et al., 1998). Por otro lado, esto demostraría la pertinencia en la promoción e impulso de este tipo de sectores en la economía.

Se ha señalado que la estratificación por tamaño de empresa (micro, pequeña, mediana y grande) juega un papel importante en la definición misma de una EBT; particularmente en México, la pyme constituye más del $98 \%$ del aparato productivo. Sin embargo, hacen falta estudios que den cuenta de su aportación al desempeño de los sectores de base tecnológica. No obstante, en las últimas décadas se ha destacado el papel de la pyme de base tecnológica en la creación de empleo y el crecimiento económico (Rae, 2006; Ganotakis y Love, 2011; Li et al., 2012), así como la derrama tecnológica (Tsvetkova et al., 2014). Es por ello que más adelante se mostrará la participación de las pymes de base tecnológica en el total de la producción.

El artículo tiene 5 secciones: en la primera se analizan las diversas definiciones de EBT y se plantea la que se usará en este artículo; en la segunda sección se expone una taxonomía de las EBT; en la tercera sección se detalla el problema que guía esta propuesta de investigación centrado en el análisis de las aportaciones que hacen las EBT por estrato de tamaño, y particularmente las pymes, a la economía nacional; en la cuarta sección se expone la metodología y la base de datos utilizada en el modelo; en la quinta sección se presentan los resultados del modelo y las estadísticas más relevantes, para finalizar con las conclusiones y los comentarios finales.

\section{Definición de empresa de base tecnológica}

Para comenzar el estudio, se propone una definición formal de una EBT, ya que esto facilitará la comprensión y los alcances del sector de base tecnológica, su comportamiento y los factores que han llevado al sector a su estado actual. En la perspectiva de la gestión estratégica se considera que una empresa es una colección de activos tanto tangibles como intangibles (Barney, 1986; Dierickx y Cool, 1989; Grant, 1991; Wernerfelt, 1984), configurados de manera tal que la organización puede generar un producto único para ser vendido en el mercado y mantener su ventaja competitiva. Por otra parte, desde la teoría económica neoclásica se considera a la empresa como una caja negra en donde entran insumos y se obtienen productos (Coase, 1937).

Cualquiera que sea la perspectiva abordada, se puede observar en las 2 definiciones anteriores que la empresa necesita de algún tipo de tecnología para poder producir. En el contexto actual de la empresa se sabe que el avance tecnológico cada vez más rápido genera nuevas oportunidades de negocio, así como nuevas necesidades en las personas. Esto ha llevado a algunas empresas a basarse en la explotación de las nuevas oportunidades de negocio derivadas del avance de la nueva tecnología (Onetti et al., 2012; Kollmer y Dowling, 2004; March y Yagüe, 1999; Granstrand, 1998; Storey y Tether, 1998a), lo cual ha dado lugar a lo que hoy llamamos EBT. Dentro de la definición anterior se entiende que aunque toda empresa tiene una base de tecnología con la cual trabaja, no toda empresa puede ser llamada EBT, ya que se requiere de una tecnología nueva; y además, esta tecnología debe generar una nueva oportunidad de negocio en los mercados.

Así mismo, una definición formal comúnmente aceptada, que será empleada en esta investigación, está constituida por empresas de no más de 25 años de edad que se basan en la explotación de una innovación o invención que implique un riesgo tecnológico sustancial (Storey y Tether, 1998a). Esta definición no solamente hace alusión a la tecnología, sino que esta tecnología debe ser reciente; y por otro lado, supone que la explotación de la oportunidad de negocio conlleva un riesgo (Onetti et al., 2012; Lockett y Wright, 2005; Kollmer y Dowling, 2004; March y Yagüe, 1999). Este riesgo es el que se asocia a las tecnologías de punta. 


\section{Taxonomía de los sectores de base tecnológica}

Uno de los fundamentos para el estudio de la EBT es definir los sectores en los cuales operan y/o su taxonomía a manera de caracterización del sector que será estudiado. En el presente apartado se realiza una aproximación a los sectores de base tecnológica en México a través de los censos económicos de 2004 y 2009, de tal forma que se puede observar la evolución del sector a través del tiempo, y en los diferentes contextos de base tecnológica. Enseguida se muestra que no se trata solo de un sector de base tecnológica, sino que estos pueden ser divididos en agrupaciones de sectores afines, tal como señalan los siguientes estudios por orden cronológico:

- Bollinger et al. (1983) estudian los sectores conformados por semiconductores, circuitos integrados, software, equipo médico y sector aeroespacial.

- Kulicke y Krupp (1987) estudian los sectores electrónicos, de computación, robótica, equipo de medición y pruebas, y la manufactura de láser.

- Autio y Yli-Renko (1998) identifican los siguientes sectores: forestal, metalúrgico, telecomunicaciones y redes de datos, electrónica, médico, biotecnológico y cuidados de salud, generación y transmisión de energía, transporte, contracción, medioambiente, comida, vestido, entre otros. Estos sectores fueron encontrados entre los principales clientes de EBT, recomendando estudiar a los clientes de las EBT como los sectores industriales que debieran ser abordados.

- En otros estudios se toma como referencia la inversión en I + D, como lo hacen Butchart (1987) y Lall (2000), donde se ha hecho una clasificación de sectores tecnológicos para describir el funcionamiento exportador de los países. Se identifican 4 sectores de acuerdo con su nivel tecnológico y se presentan de menor a mayor nivel:

1. Sectores basados en la explotación de recursos naturales.

2. Manufacturas con bajo nivel tecnológico.

3. Manufacturas con medio nivel tecnológico.

4. Manufacturas con alto nivel tecnológico.

Como se observa en los estudios de Bollinger et al. (1983) y Kulicke y Krupp (1987), la definición de un sector tecnológico se basa en los sectores que en el momento específico significaron alguna novedad, pero sin tener establecido aún un consenso sobre las características propias de un sector de base tecnológica.

La principal limitación del estudio de Autio y Yli-Renko (1998) es que los sectores de base tecnológica se clasifican de acuerdo con los clientes de estas empresas, pero es complicado observar a simple vista un cliente de una EBT, además de que no todos los clientes de estas empresas son EBT, ya que algunos son consumidores finales, personas o familias.

Por otro lado, en las definiciones de Butchart (1987) y Lall (2000), la principal limitación con este sistema de clasificación radica en el hecho de aludir solo a los niveles de avance tecnológico de las máquinas y su nivel de sofisticación. Sin embargo, las manufacturas con alto nivel tecnológico de Butchart y Lall no necesariamente son manufacturas de EBT, ya que no necesariamente estas provienen de nuevas tecnologías que aprovechan oportunidades de negocios nuevos.

Otros estudios definen los sectores de base tecnológica como: los sectores de telecomunicaciones, hardware, software, Internet, semiconductores y algunos equipos electrónicos relacionados, biotecnologías, tecnologías médicas, química y farmacéutica, instrumentos de medición y sistemas láser, nanotecnología, microelectrónica y aeroespacial (Tsvetkova et al., 2014; Oakey, Groen, van der Sijde y Cook, 1993; Onetti et al., 2012; Li et al., 2012; Kollmer y Dowling, 2004; Fontes 
y Coombs, 2001). En la mayor parte de los casos, estas taxonomías coinciden con las definiciones de Autio et al. (1998). Sin embargo, estos sectores deben ser contextualizados dadas las diferencias culturales y económicas de cada país, y la intensidad tecnológica de cada sector. Estos factores tienen un papel importante, como señalan algunos autores (Butchart, 1987; Storey y Tether, 1998a; Lall, 2000).

A nivel nacional se identificaron programas de apoyo a la EBT desde 1992. Uno de los más importantes ha sido el Programa de Incubadoras de Empresas de Base Tecnológica. También se establecieron otros programas de financiamiento, como el Fondo de Innovación Tecnológica, el Fondo PYME y el Programa Nacional de Software. No es hasta 2013 cuando el Gobierno de México, a través de la Secretaría de Economía y el Programa para el Desarrollo de Industrias de Alta Tecnología (PRODIAT), define los sectores de base tecnológica y establece apoyos económicos para las empresas de las siguientes ramas de clasificación industrial del SCIAN:

- 333: fabricación de maquinaria y equipo.

- 334: fabricación de equipo de computación, comunicación, medición y otros equipos, componentes y otros equipos electrónicos.

- 335: fabricación de equipo de generación eléctrica y aparatos y accesorios eléctricos.

- 336: fabricación de equipo de transporte y partes para vehículos automotores.

Sin embargo, la clasificación del PRODIAT carece de otros sectores de base tecnológica incluidos por Autio et al. (1998) y Oakey et al. (1993). Tampoco desglosa dentro de las ramas de actividad los subsectores de base tecnológica.

Por otro lado, a nivel estatal se han definido sectores de base tecnológica entre los que se encuentran los siguientes:

- Jalisco tiene el Programa Especial de Ciencia y Tecnología 2008-2012, donde se especifica que son 18 las áreas estratégicas que se pretende fomentar. Las áreas que coinciden con las del PRODIAT son: biotecnologías, medicina, tecnologías de la información y telecomunicaciones, eléctrica y electrónica, farmacéutica y ciencias de la salud, química y petroquímica.

- Nuevo León decretó la Ley de impulso a la innovación tecnológica del año 2010. Esta ley define como sectores de base tecnológica prioritarios, mas no limitativos, los siguientes: biotecnologías, mecatrónica, tecnologías de la información y comunicación, salud, nanotecnología y manufacturas avanzadas.

- En el Distrito Federal, en el Programa de Ciencia y Tecnología 2007-2012 se establecen como sectores tecnológicos los sectores tecnológicos prioritarios, que son los siguientes: tecnologías urbanas, salud, biotecnologías y tecnologías de medio ambiente. Tomando en cuenta las taxonomías previas y los estudios anteriores, se identifican a través del SCIAN y del INEGI los sectores de base tecnológica considerados en este trabajo.

Tomando como referencia algunas similitudes o agrupaciones de los estudios señalados a nivel nacional e internacional, podemos clasificar los sectores de base tecnológica en 4:

1. Telecomunicaciones y tecnologías de la información.

2. Electrónica y fabricación de equipo vehicular.

3. Biotecnologías y tecnología médica.

4. Química y farmacéutica. 
Esta clasificación será la base de este trabajo y su descripción amplia está en las siguientes secciones. Cabe señalar que no se toma la definición del PRODIAT como los sectores de base tecnológica ya que se limita algunos sectores, pero no a la totalidad señalada en la literatura revisada. Además, no todos los sectores señalados por el PRODIAT se pueden considerar EBT, pues existen pymes que en el caso mexicano se clasifican en los sectores descritos por el SCIAN pero no son propiamente una EBT, dado que en este artículo se toma como EBT la definición propuesta por Storey y Tether (1998a), donde se alude a la tecnología de punta, la edad de las empresas y la oportunidad de negocio que genera esta tecnología como principales características de este tipo de empresa.

\section{Planteamiento del problema}

El propósito de este trabajo es mostrar los aportes de la EBT por estrato de tamaño; en particular, mostrar los aportes de la pyme de base tecnológica al total de los sectores de base tecnológica en el contexto mexicano, y analizar cuál es el impacto de estos sectores en la economía nacional. Adicionalmente se planteó la necesidad de reconocer si las EBT funcionan con economías de escala, ya que esto demostraría que este tipo de empresas no necesariamente deben alcanzar una escala de producción óptima para lograr el éxito. Por otro lado, se tiene que las EBT son empresas intensivas en el uso de conocimiento y generan mayores volúmenes de valor agregado. Por esta razón, en este artículo se plantea el cociente de la generación de valor agregado por cada unidad de producción bruta, ya que esto mostrará la eficiencia productiva en términos de generación de valor agregado por cada unidad de producción. Por lo anterior, se plantean 2 hipótesis de investigación:

- Las EBT de tamaño pequeño generan un valor agregado equiparable al de la EBT de tamaño grande, por lo que no requieren de rendimientos crecientes para lograr el éxito de mercado.

- El tamaño de la EBT está positivamente relacionado con su nivel tecnológico.

Estas hipótesis de investigación evidencian que no es necesariamente la EBT de tamaño grande la que tiene mayores niveles tecnológicos, como podría pensarse dada su capacidad financiera y económica. Por otro lado, la flexibilidad de la EBT pequeña le permite generar montos de valor agregado equiparables a los de la gran empresa, al especializarse en nichos de mercado que le permiten generar este valor agregado a sus clientes. Finalmente, se destaca que la EBT no requiere de rendimientos crecientes de escala para alcanzar grandes volúmenes de producción que le representen el éxito en el mercado.

\section{Metodología y base de datos}

Se parte de los 4 sectores definidos como sectores de base tecnológica que incorporan la clasificación de empresas que hace el SCIAN dentro del INEGI como base para identificar las EBT dentro del contexto mexicano. Se consideran los censos económicos de 2004 y 2009 por contener la información más reciente que se tiene disponible. A partir de estos datos se hace una descripción de la evolución que ha seguido la EBT, así como una inferencia sobre la forma en la que trabajan estas empresas.

De hecho, no es nuevo utilizar el SCIAN para identificar los sectores de base tecnológica, ya que en otros estudios (Tsvetkova et al., 2014) se utiliza este sistema de clasificación para identificar los sectores de cómputo, comunicación, audio, vídeo, equipo de navegación y equipo 
óptico. Sin embargo, este estudio parte, como se ha señalado, de los 4 sectores de base tecnológica anteriormente descritos.

\section{Caracterización de los sectores de base tecnológica}

De acuerdo con la clasificación SCIAN que maneja INEGI, y según las definiciones dadas anteriormente, se clasifican los sectores de base tecnológica en la tabla 1. En dicha tabla se muestran las 15 actividades que constituyen los sectores de telecomunicaciones y tecnologías de la información dentro del SCIAN. Se han excluido las ramas 5181, 5171 y 5172, ya que se refieren a proveedores de Internet y servicios telefónicos, entendiendo que se trata solo de la comercialización de estos servicios por parte de los grandes monopolios del país.

En la tabla 1 se muestran las 22 actividades que se identifican dentro del SCIAN para estratificar el sector de electrónica y fabricación de equipo vehicular, dentro de la clasificación del SCIAN. Las subramas 3361 y 3363 tendrán un tratamiento especial, principalmente las empresas de tamaño grande, ya que se trata de las grandes armadoras automotrices del país, las cuales no contribuyen de manera significativa al sector de base tecnológica aquí descrito. Se muestran las 6 actividades que describen el sector de biotecnologías y tecnologías médicas dentro de la clasificación del SCIAN. Se detallan las 10 actividades que conforman el sector de química y farmacéutica.

De esta manera, se desagregan los sectores industriales de base tecnológica en México, identificando 4 grandes sectores:

1. Telecomunicaciones y tecnologías de la información.

2. Electrónica y fabricación de equipo vehicular.

3. Biotecnologías y tecnologías médicas.

4. Química y farmacéutica.

En secciones posteriores, se hará uso de esta clasificación para describir tanto el mercado como la dinámica que sigue la industria de base tecnológica en México. Por otra parte, la metodología propuesta para analizar si una EBT de tamaño pequeño genera mayores volúmenes de valor agregado es utilizando una razón de valor agregado a producción como VA/PRODUCCIÓN. Esto permitirá identificar si las EBT de tamaño pequeño generan volúmenes de valor agregado equiparable al de las EBT de tamaño grande. Para ello se tomarán en cuenta la producción bruta total y el valor agregado censal bruto, ya que ambos están contenidos dentro de los censos económicos de 2004 y 2009.

\section{Modelo de regresión}

Existen otros estudios en donde se han manejado los modelos de regresión para estimar la productividad factorial. Como trabajo pionero se encuentra el de Solow (1957), en donde se analiza el cambio técnico de factores a través de las funciones de producción, localizando de esta manera las economías de escala y la productividad factorial total. En el presente estudio se aproxima la estimación mediante una función de producción tipo Cobb-Douglas con datos recabados de los censos económicos de 2004 y 2009. Sin embargo, es importante señalar que en otros estudios sobre productividad en la EBT se han realizado mediante el análisis de regresión para describir la productividad de la EBT, pero no para hacer comparativos de la EBT en sus diferentes sectores (Yagüe y Mach, 2013; Li et al., 2012; Ganotakis y Love, 2011), excepto Ortin y Vendrell (2014), quienes comparan la EBT con las spin-off. 
Tabla 1

Sectores de base tecnológica

Rama Concepto

Sector de telecomunicaciones y tecnologías de la información

$3341 \quad$ Fabricación de ordenadores y equipo periférico

$3342 \quad$ Fabricación de equipo de comunicación

$3343 \quad$ Fabricación de equipo de audio y de vídeo

$3344 \quad$ Fabricación de componentes electrónicos

$3346 \quad$ Fabricación y reproducción de medios magnéticos y ópticos

5112 Edición de software y edición de software integrada con la reproducción

$5121 \quad$ Industria fílmica y del vídeo

$5122 \quad$ Industria del sonido

$5151 \quad$ Transmisión de programas de radio y televisión

$5152 \quad$ Producción de programación de canales para sistemas de televisión por cable o por satélite

$5161 \quad$ Creación y difusión de contenido exclusivamente a través de Internet

$5174 \quad$ Servicios de telecomunicaciones por satélite

$5179 \quad$ Otros servicios de telecomunicaciones

5182 Procesamiento electrónico de información, hospedaje y otros servicios relacionados

$5191 \quad$ Otros servicios de información

Sector de electrónica y fabricación de equipo vehicular

$3331 \quad$ Fabricación de maquinaria y equipo agropecuario para la construcción y para la industria extractiva

3332 Fabricación de maquinaria y equipo para las industrias manufactureras, excepto la metalmecánica

3333 Fabricación de maquinaria y equipo para el comercio y los servicios

3334 Fabricación de equipo de aire acondicionado, calefacción y de refrigeración industrial y comercial

$3335 \quad$ Fabricación de maquinaria y equipo para la industria metalmecánica

3336 Fabricación de motores de combustión interna, turbinas y transmisiones

3339 Fabricación de otra maquinaria y equipo para la industria en general

3345 Fabricación de instrumentos de medición, control, navegación y equipo médico electrónico

$3351 \quad$ Fabricación de accesorios de iluminación

$3352 \quad$ Fabricación de aparatos eléctricos de uso doméstico

3353 Fabricación de equipo de generación y distribución de energía eléctrica

$3359 \quad$ Fabricación de otros equipos y accesorios eléctricos

$3361 \quad$ Fabricación de automóviles y camiones

$3362 \quad$ Fabricación de carrocerías y remolques

$3363 \quad$ Fabricación de partes para vehículos automotores

$3364 \quad$ Fabricación de equipo aeroespacial

3365 Fabricación de equipo ferroviario

$3366 \quad$ Fabricación de embarcaciones

$3369 \quad$ Fabricación de otro equipo de transporte

5415 Servicios de diseño de sistemas de cómputo y servicios relacionados

8112 Reparación y mantenimiento de equipo electrónico y de equipo de precisión

8113 Reparación y mantenimiento de maquinaria y equipo agropecuario, industrial, comercial y de servicios

Sector de biotecnologías y tecnologías médicas

$3111 \quad$ Elaboración de alimentos para animales

3119 Otras industrias alimentarias

6214 Centros para la atención de pacientes que no requieren hospitalización

$6215 \quad$ Laboratorios médicos y de diagnóstico

6219 Servicios de ambulancias, de bancos de órganos y otros servicios auxiliares al tratamiento médico

$6223 \quad$ Hospitales de otras especialidades médicas

Sector de química y farmacéutica

$3241 \quad$ Fabricación de productos derivados del petróleo y del carbón

$3251 \quad$ Fabricación de productos químicos básicos

3252 Fabricación de resinas y hules sintéticos, y fibras químicas 
Tabla 1 (continuación)

\begin{tabular}{ll}
\hline Rama & Concepto \\
\hline 3253 & Fabricación de fertilizantes, pesticidas y otros agroquímicos \\
3254 & Fabricación de productos farmacéuticos \\
3255 & Fabricación de pinturas, recubrimientos y adhesivos \\
3256 & Fabricación de jabones, limpiadores y preparaciones de tocador \\
3259 & Fabricación de otros productos químicos \\
3261 & Fabricación de productos de plástico \\
3262 & Fabricación de productos de hule
\end{tabular}

Fuente: Elaboración propia a partir del Sistema de Clasificación Industrial de América del Norte (2007).

Esta estimación permitirá definir si en los sectores tecnológicos existen economías con rendimientos de escala creciente, ya que una de las hipótesis principales es la existencia de rendimientos crecientes en los sectores de base tecnológica. El modelo econométrico planteado es el siguiente:

$$
\log (\mathrm{Y})=\beta 1 * \text { Tamaño }+\beta 1 *[\log (\mathrm{L}) * \operatorname{Sector}]+\beta 1 * *[\log (\mathrm{K}) * \operatorname{Sector}]+\mathrm{e}
$$

Donde « $\log (\mathrm{Y}) »$ representa el logaritmo de la producción, «Log(L)» representa el logaritmo del trabajo, «Log $(\mathrm{K}) »$ representa el logaritmo del capital, «Sector» es una variable dicotómica que representa el sector tecnológico, y «Tamaño» es una variable dicotómica que representa el tamaño de la empresa. Este modelo econométrico permitirá verificar si existen economías de escala debido a la siguiente condición: si $(\beta 2+\beta 3)$ es igual a uno se dice que existen economías de escala; si la condición es mayor a uno entonces existen economías crecientes de escala; cualquiera de los 2 resultados mostraría que existe una eficiencia relativa en el uso de los factores en los sectores tecnológicos.

Sin embargo, primero se deben definir las variables que se utilizarán en el presente estudio. Se tienen distintas variables Proxy para la producción, el trabajo y el capital, ya que existen variables estáticas y dinámicas ${ }^{3}$. Para la producción se tiene como variable estática el producto bruto total, y como variable dinámica, el valor agregado censal bruto. Para el trabajo se tiene como variable estática el personal ocupado, y como variable dinámica, las horas trabajadas, y finalmente, para el capital se tiene como variable estática el acervo total de capital fijo, y como variable dinámica, la formación bruta de capital fijo. Esta información se ve reflejada en la tabla 2.

Como se puede apreciar, existen 2 variables Proxy para la producción y 2 variables Proxy para las variables explicativas, y por otra parte, se tienen 2 censos económicos (2004 y 2009). Derivado de lo anterior, se mostrarán los resultados de las regresiones para cada una de las combinaciones de análisis de regresión múltiple y para cada uno de los censos económicos. Este análisis de regresión permitirá contrastar las hipótesis 2 y 3 presentadas en el planteamiento del problema.

Las unidades que se tomarán en cuenta para la medición son empresas representativas para cada estrato de tamaño de empresa. De esta manera se puede acceder a las bases de datos a través de

\footnotetext{
${ }^{3}$ La producción, el personal ocupado y el acervo de activo fijo representan variables estáticas debido a que en su definición cambian con relativa lentitud; un ejemplo de ello es el acervo de activo fijo, pues no todo el activo fijo es utilizado en los procesos de producción. Como contraparte, se tienen como variables dinámicas el valor agregado censal bruto y las horas de trabajo y formación bruta de capital fijo, que cambian con mayor dinamismo ante cambios en el proceso productivo. Siguiendo el ejemplo antes señalado, la formación bruta de capital fijo muestra con mayor detalle el cambio en la inversión debido a procesos productivos. Ambos ejemplos ponen de manifiesto el papel de los activos fijos en el proceso productivo; la única diferencia es su dinamismo como variable explicativa.
} 
Tabla 2

Descripción de las variables utilizadas en el modelo econométrico

\begin{tabular}{|c|c|c|c|}
\hline Variables & Descripción & Escala de la variable & Tipo de variable \\
\hline LOG(PROD) & Logaritmo de la producción bruta total & Números reales & Estática \\
\hline LOG(VA) & Logaritmo del valor agregado censal bruto & Números reales & Dinámica \\
\hline LOG(HORAS) & Logaritmo de las horas trabajadas & Números reales & Dinámica \\
\hline $\mathrm{LOG}(\mathrm{FBCF})$ & Logaritmo de la formación bruta de capital fijo & Números reales & Dinámica \\
\hline $\mathrm{LOG}(\mathrm{PO})$ & Logaritmo del personal ocupado & Números reales & Estática \\
\hline LOG(ACERVO) & Logaritmo del acervo total de capital fijo & Números reales & Estática \\
\hline SEC1 & $\begin{array}{l}\text { Sector de telecomunicaciones y tecnologías de } \\
\text { la información }\end{array}$ & $\begin{array}{l}1 \text { si pertenece al sector y } 0 \\
\text { en otro caso }\end{array}$ & Interacción \\
\hline SEC2 & Sector de electrónica y tecnología vehicular & $\begin{array}{l}1 \text { si pertenece al sector y } 0 \\
\text { en otro caso }\end{array}$ & Interacción \\
\hline SEC3 & Sector de biotecnologías y tecnologías médicas & $\begin{array}{l}1 \text { si pertenece al sector y } 0 \\
\text { en otro caso }\end{array}$ & Interacción \\
\hline SEC4 & Sector de química y farmacéutica & $\begin{array}{l}1 \text { si pertenece al sector y } 0 \\
\text { en otro caso }\end{array}$ & Interacción \\
\hline TAM1 & Tamaño de microempresa & $\begin{array}{l}1 \text { si pertenece al estrato y } \\
0 \text { en otro caso }\end{array}$ & Control \\
\hline TAM2 & Tamaño de pequeña empresa & $\begin{array}{l}1 \text { si pertenece al estrato y } \\
0 \text { en otro caso }\end{array}$ & Control \\
\hline TAM3 & Tamaño de mediana empresa & $\begin{array}{l}1 \text { si pertenece al estrato y } \\
0 \text { en otro caso }\end{array}$ & Control \\
\hline TAM4 & Tamaño de gran empresa & $\begin{array}{l}1 \text { si pertenece al estrato y } \\
0 \text { en otro caso }\end{array}$ & Control \\
\hline
\end{tabular}

Elaborada con base en información de censos económicos (2004 y 2009), Instituto Nacional de Estadística y Geografía (2009).

INEGI y los censos económicos, con clasificaciones por número de empleados, como se muestra en la tabla 3.

De esta forma, en la tabla 3 se observa que cada estrato representa una empresa con un tamaño específico, la cual puede ser clasificada como micro, pequeña, mediana o grande. Para cada una

Tabla 3

Clasificación de empresas por número de empleados y estrato de tamaño

\begin{tabular}{ll}
\hline Número de empleados & Estrato de empresa \\
\hline De 0 a 2 & Micro \\
De 3 a 5 & Micro \\
De 6 a 10 & Micro \\
De 11 a 15 & Pequeña \\
De 16 a 20 & Pequeña \\
De 21 a 30 & Pequeña \\
De 31 a 50 & Pequeña \\
De 51 a 100 & Mediana \\
De 101 a 250 & Mediana \\
De 251 a 500 & Grande \\
De 501 a 1,000 & Grande \\
De 1,001 y más & Grande
\end{tabular}

Elaboración propia a partir de censos económicos (2004 y 2009), Instituto Nacional de Estadística y Geografía (2009). 
Tabla 4

Contribución de los sectores tecnológicos en el total nacional en censo económico 2004

\begin{tabular}{lllcc}
\hline Sector tecnológico & $\begin{array}{l}\text { Unidades } \\
\text { económicas }\end{array}$ & $\begin{array}{l}\text { Personal } \\
\text { ocupado total }\end{array}$ & $\begin{array}{l}\text { Producción bruta total } \\
\text { (miles de pesos) }\end{array}$ & $\begin{array}{l}\text { Valor agregado } \\
\text { censal bruto } \\
\text { (miles de pesos) }\end{array}$ \\
\hline Telecomunicaciones y TIC & 4,053 & 333,475 & $175,790,824$ & $72,832,763$ \\
Electrónica y equipo vehicular & 37,320 & 466,432 & $212,196,024$ & $85,584,047$ \\
Biotecnología y tecnología médica & 11,726 & 123,461 & $94,080,377$ & $27,415,169$ \\
Química y farmacéutica & 7,714 & 460,682 & $728,221,012$ & $214,057,389$ \\
Total sectores tecnológicos & 60,813 & $1,384,050$ & $1,210,288,237$ & $399,889,368$ \\
Total nacional & $3,002,720$ & $16,018,201$ & $5,974,656,821$ & $3,048,348,987$ \\
Porcentaje respecto del nacional & 2.03 & 8.64 & 20.26 & 13.12 \\
\hline
\end{tabular}

Fuente: Elaboración propia a partir de censos económicos (2004).

de estas empresas representativas se toman en consideración las variables expresadas en la tabla 2. Esta base de datos cuenta con un total de 598 observaciones y es la base de datos que servirá para ejecutar el análisis de regresión.

\section{Resultados y estadísticas relevantes}

A continuación, con el objetivo de entender el comportamiento y las pautas que ha seguido el sector de base tecnológica en México, se muestra la participación de los sectores tecnológicos a la economía nacional. En la tabla 4 se observa que no es menor la contribución de las EBT al total de la producción bruta nacional en 2004. A pesar de ser solo el $2.03 \%$ del total de las empresas, estas generan el $20.26 \%$ de la producción bruta nacional, con el $8.64 \%$ del personal ocupado y generando un valor agregado del $13.12 \%$.

En la tabla 5 se observa que no es menor la participación de las EBT al total de la producción bruta nacional en 2009. A pesar de ser solo el $2.05 \%$ del total de las empresas, estas generan el $22.09 \%$ de la producción bruta nacional, con el $8.27 \%$ del personal ocupado y aportando un valor agregado del $12.99 \%$.

Como se puede observar, los datos de las EBT se han mantenido constantes, a excepción de los relacionados con el total de personal ocupado. Este resultado puede señalar que existe una menor cantidad de personal ocupado: $8.27 \%$. Por otra parte, haciendo una descripción por tamaños de

Tabla 5

Contribución de los sectores tecnológicos en el total nacional en censo económico 2009

\begin{tabular}{lcccc}
\hline Sector tecnológico & $\begin{array}{l}\text { Unidades } \\
\text { económicas }\end{array}$ & $\begin{array}{l}\text { Personal } \\
\text { ocupado total }\end{array}$ & $\begin{array}{l}\text { Producción bruta total } \\
\text { (miles de pesos) }\end{array}$ & $\begin{array}{l}\text { Valor agregado } \\
\text { censal bruto } \\
\text { (miles de pesos) }\end{array}$ \\
\hline Telecomunicaciones y TIC & 7,152 & 398,028 & $190,041,348$ & $80,436,227$ \\
Electrónica y equipo vehicular & 41,484 & 567,393 & $384,170,989$ & $149,863,344$ \\
Biotecnología y tecnología médica & 18,384 & 177,860 & $180,937,658$ & $52,945,613$ \\
Química y farmacéutica & 9,222 & 500,515 & $1,559,858,902$ & $338,517,362$ \\
Total sectores tecnológicos & 76,242 & $1,643,796$ & $2,315,008,897$ & $621,762,546$ \\
Total nacional & $3,721,430$ & $19,881,146$ & $10,480,980,684$ & $4,785,819,527$ \\
Porcentaje respecto del nacional & 2.05 & 8.27 & 22.09 & 12.99 \\
\hline
\end{tabular}

Fuente: Elaboración propia a partir de censos económicos (2009). 
empresa en los mismos sectores tecnológicos, tenemos los datos que se presentan en la tabla 9. Estos datos muestran qué tanto aportan los diferentes estratos de empresa al total de la producción de EBT.

En la tabla 6 se puede apreciar que alrededor del $80 \%$ de las empresas son de tamaño micro. Estas empresas generan el $2.36 \%$ de la producción total. Las empresas grandes representan solamente el $1.8 \%$ del total de empresas participantes, pero generan el $73.7 \%$ del total de la producción de los sectores de base tecnológica. Sin embargo, se puede apreciar que la microempresa genera un mayor volumen de valor agregado en relación con el total de lo que produce, ya que la razón valor/producción es la más alta, con un $44.76 \%$. Este resultado puede ser un indicador de las ventajas de la flexibilidad del tamaño de la empresa en estos sectores.

En la tabla 7 se puede apreciar que alrededor del $83.5 \%$ de las empresas son de tamaño micro y generan solamente el $1.43 \%$ de la producción total. Las empresas grandes representan solamente el $1.63 \%$ del total de empresas y generan el $76.8 \%$ del total de la producción de los sectores de base tecnológica. Sin embargo, se puede apreciar que la microempresa genera un mayor volumen de valor agregado en relación con el total de lo que produce, ya que la razón valor/producción es la más alta, con un $36.99 \%$. Este resultado puede evidenciar, como en el caso anterior, que el tamaño de la empresa está asociado a una mayor flexibilidad en estos sectores, aunque con relación a 2004 se ha reducido de manera considerable. Es necesario señalar, además, que la EBT de tamaño grande ha disminuido su cociente de valor agregado/producción de manera considerable, y aunque la microempresa también disminuyó su cociente, esta se mantiene con un cociente claramente superior al del resto de los estratos de tamaño. Estos hallazgos muestran que para este tipo de empresas no es necesaria una escala de producción amplia, en términos de lograr la efectiva generación de valor agregado por unidad producida, es decir, las microempresas entregan mayor valor a sus clientes por unidad de producto dado el cociente señalado, lo cual abre puertas para otro tipo de investigaciones.

Hasta este punto, se puede observar que aunque se trata de alrededor del $2 \%$ del total de empresas, estas generan alrededor del $20 \%$ del total de la producción de industrias manufactureras del país. Sin embargo, a pesar de ser en su gran mayoría empresas de tamaño micro y pequeñas, las empresas que generan la mayor parte de la producción en sectores de base tecnológica son las empresas de tamaño grande. No obstante, es necesario señalar también que la razón de valor/producción muestra que la flexibilidad de la empresa micro y pequeña les ayuda a generar mayores volúmenes de valor agregado en relación con la mediana y gran empresa.

Estos datos muestran que las empresas de tamaño micro generan de manera relativa mayores volúmenes de valor agregado que las empresas de tamaño grande. Estos resultados se sostienen tanto para los censos de 2004 como para los censos de 2009, por lo que se considera que los resultados son consistentes.

\section{Resultados del análisis de regresión múltiple para censos económicos de 2004}

Se puede observar en la tabla 8 que en la mayor parte de las regresiones existen rendimientos crecientes de escala. Por ejemplo, sumando los coeficientes LOG(HORAS)*SEC1 + LOG $(\mathrm{FBCF}) *$ SEC1 de la primera regresión $(0.87+0.15=1.02)$ se observan rendimientos crecientes de escala, los cuales ya fueron contrastados con una prueba de Wald con resultados significativos. Esto implica que en el año del censo económico se puede decir que estos sectores trabajaron con rendimientos crecientes de escala, con excepción del sector 3 de biotecnologías y tecnología médica, ya que solo en una de las regresiones se encontraron rendimientos crecientes. Por otra parte, en todas las regresiones se estima que el nivel tecnológico con el que parten las 
Tabla 6

Participación por tamaño de empresa a los sectores tecnológicos en 2004

\begin{tabular}{|c|c|c|c|c|c|c|c|}
\hline $\begin{array}{l}\text { Tamaño de } \\
\text { la empresa }\end{array}$ & $\begin{array}{l}\text { Unidades } \\
\text { económicas }\end{array}$ & $\begin{array}{l}\text { Personal } \\
\text { ocupado total }\end{array}$ & $\begin{array}{l}\text { Producción bruta } \\
\text { total (miles de } \\
\text { pesos) }\end{array}$ & $\begin{array}{l}\text { Valor agregado } \\
\text { censal bruto } \\
\text { (miles de pesos) }\end{array}$ & $\begin{array}{l}\text { Formación bruta de } \\
\text { capital fijo (miles } \\
\text { de pesos) }\end{array}$ & $\begin{array}{l}\text { Acervo total de } \\
\text { activos fijos } \\
\text { (miles de pesos) }\end{array}$ & $\begin{array}{l}\text { Razón de } \\
\text { valor/ } \\
\text { producción }\end{array}$ \\
\hline Micro & 49,808 & 133,120 & $28,575,840$ & $12,789,478$ & 608,084 & $16,946,732$ & 0.4476 \\
\hline Pequeña & 7,024 & 155,926 & $73,212,324$ & $23,684,817$ & $2,643,332$ & $30,708,423$ & 0.3235 \\
\hline Mediana & 2,888 & 324,616 & $216,485,592$ & $70,662,484$ & $6,008,532$ & $94,265,891$ & 0.3264 \\
\hline Grande & 1,093 & 770,388 & $892,014,481$ & $292,752,589$ & $21,383,386$ & $334,082,284$ & 0.3282 \\
\hline Total & 60,813 & $1,384,050$ & $1,210,288,237$ & $399,889,368$ & $30,643,334$ & $476,003,330$ & 0.3304 \\
\hline
\end{tabular}

Fuente: Elaboración propia a partir de censos económicos (2004). 
Tabla 7

Participación por tamaño de empresa a los sectores tecnológicos en 2009

\begin{tabular}{lcllccc}
\hline $\begin{array}{l}\text { Tamaño de } \\
\text { la empresa }\end{array}$ & $\begin{array}{l}\text { Unidades } \\
\text { económicas }\end{array}$ & $\begin{array}{l}\text { Personal } \\
\text { ocupado total }\end{array}$ & $\begin{array}{l}\text { Producción bruta } \\
\text { total (miles de } \\
\text { pesos) }\end{array}$ & $\begin{array}{l}\text { Valor agregado } \\
\text { censal bruto } \\
\text { (miles de pesos) }\end{array}$ & $\begin{array}{l}\text { Formación bruta de } \\
\text { capital fijo (miles } \\
\text { de pesos) }\end{array}$ & $\begin{array}{l}\text { Acervo total de } \\
\text { activos fijos } \\
\text { (miles de pesos) }\end{array}$ \\
\hline Micro & 63,661 & 174,241 & $33,069,053$ & $12,231,595$ & 660,766 & $\begin{array}{l}\text { Razón de } \\
\text { valor/ } \\
\text { producción }\end{array}$ \\
Pequeña & 8,239 & 180,520 & $114,162,625$ & $37,567,205$ & $2,099,621$ & $20,029,803$ \\
Mediana & 3,101 & 350,395 & $390,129,986$ & $120,723,941$ & $8,923,668$ & 0.3699 \\
Grande & 1,241 & 938,640 & $1,777,647,233$ & $451,239,805$ & $27,676,126$ & $121,284,722$ \\
Total & 76,242 & $1,643,796$ & $2,315,008,897$ & $621,762,546$ & $39,360,181$ & $504,413,923$ \\
\end{tabular}

Fuente: Elaboración propia a partir de censos económicos (2009). 
Tabla 8

Resultados del análisis de regresión múltiple para censos económicos en 2004

\begin{tabular}{|c|c|c|c|c|c|c|c|c|}
\hline \multirow[t]{2}{*}{ Variable dependiente } & \multicolumn{2}{|c|}{ LOG(PROD) } & \multicolumn{2}{|c|}{ LOG(VA) } & \multicolumn{2}{|c|}{ LOG(PROD) } & \multicolumn{2}{|c|}{ LOG(VA) } \\
\hline & Coeficiente & $\mathrm{DE}$ & Coeficiente & $\mathrm{DE}$ & Coeficiente & $\mathrm{DE}$ & Coeficiente & DE \\
\hline LOG(HORAS)*SEC1 & 0.87 & $0.05^{\mathrm{a}}$ & 0.9 & $0.06^{\mathrm{a}}$ & & & & \\
\hline LOG(FBCF)*SEC1 & 0.15 & $0.04^{\mathrm{a}}$ & 0.17 & $0.05^{\mathrm{a}}$ & & & & \\
\hline LOG(HORAS)*SEC2 & 0.85 & $0.06^{\mathrm{a}}$ & 0.91 & $0.06^{\mathrm{a}}$ & & & & \\
\hline LOG(FBCF)*SEC2 & 0.16 & $0.04^{\mathrm{a}}$ & 0.14 & $0.05^{\mathrm{a}}$ & & & & \\
\hline LOG(HORAS)*SEC3 & 0.11 & 0.12 & 0.31 & $0.13^{b}$ & & & & \\
\hline LOG(FBCF)*SEC3 & 0.79 & $0.10^{\mathrm{a}}$ & 0.64 & $0.11^{\mathrm{a}}$ & & & & \\
\hline LOG(HORAS)*SEC4 & 0.6 & $0.07^{\mathrm{a}}$ & 0.63 & $0.11^{\mathrm{a}}$ & & & & \\
\hline LOG(FBCF)*SEC4 & 0.42 & $0.05^{\mathrm{a}}$ & 0.4 & $0.08^{\mathrm{a}}$ & & & & \\
\hline $\mathrm{LOG}(\mathrm{PO}) * \mathrm{SEC} 1$ & & & & & 0.65 & $0.09^{\mathrm{a}}$ & 0.59 & $0.09^{\mathrm{a}}$ \\
\hline LOG(ACERVO)*SEC1 & & & & & 0.4 & $0.07^{\mathrm{a}}$ & 0.44 & $0.07^{\mathrm{a}}$ \\
\hline $\mathrm{LOG}(\mathrm{PO}) * \mathrm{SEC} 2$ & & & & & 0.73 & $0.09^{\mathrm{a}}$ & 0.77 & $0.09^{\mathrm{a}}$ \\
\hline LOG(ACERVO)*SEC2 & & & & & 0.35 & $0.08^{\mathrm{a}}$ & 0.33 & $0.08^{\mathrm{a}}$ \\
\hline LOG(PO)*SEC3 & & & & & 0.15 & 0.3 & 0.81 & $0.20^{\mathrm{a}}$ \\
\hline LOG(ACERVO)*SEC3 & & & & & 0.68 & $0.18^{\mathrm{a}}$ & 0.28 & $0.13^{\mathrm{b}}$ \\
\hline LOG(PO)*SEC4 & & & & & 0.46 & $0.10^{\mathrm{a}}$ & 0.54 & $0.14^{\mathrm{a}}$ \\
\hline LOG(ACERVO)*SEC4 & & & & & 0.53 & $0.07^{\mathrm{a}}$ & 0.47 & $0.09^{\mathrm{a}}$ \\
\hline TAM1 & 4.27 & $0.21^{\mathrm{a}}$ & 3.07 & $0.26^{\mathrm{a}}$ & 3.16 & $0.38^{\mathrm{a}}$ & 2.26 & $0.40^{\mathrm{a}}$ \\
\hline TAM2 & 4.65 & $0.21^{\mathrm{a}}$ & 3.45 & $0.25^{\mathrm{a}}$ & 3.6 & $0.36^{\mathrm{a}}$ & 2.69 & $0.39^{\mathrm{a}}$ \\
\hline TAM3 & 4.85 & $0.27^{\mathrm{a}}$ & 3.57 & $0.32^{\mathrm{a}}$ & 3.69 & $0.42^{\mathrm{a}}$ & 2.74 & $0.45^{\mathrm{a}}$ \\
\hline TAM4 & 4.96 & $0.28^{\mathrm{a}}$ & 3.76 & $0.33^{\mathrm{a}}$ & 3.82 & $0.44^{\mathrm{a}}$ & 2.97 & $0.46^{\mathrm{a}}$ \\
\hline $\mathrm{R} 2$ & & 0.91 & & 0.87 & & 0.92 & & 0.88 \\
\hline R2 ajustado & & 0.91 & & 0.87 & & 0.92 & & 0.88 \\
\hline EE de la regresión & & 0.65 & & 0.79 & & 0.67 & & 0.8 \\
\hline Suma de residuales & & 212 & & 295 & & 251 & & 340 \\
\hline Log likelihood & & -499 & & -568 & & -572 & & -640 \\
\hline Observaciones & & 564 & & 538 & & 564 & & 538 \\
\hline
\end{tabular}

Estimación de la función de producción tipo Cobb-Douglas por sector industrial de base tecnológica y con variables dicotómicas de tamaño de empresa. En las primeras 4 columnas se toman variables explicativas estáticas y en las columnas 5 a 8 se toman variables dinámicas. Se muestran los coeficientes de regresión y los errores estándar. Las regresiones fueron ejecutadas con errores consistentes de White para mitigar problemas de heterocedasticidad.

${ }^{\text {a }}$ El coeficiente es significativo al $1 \%$.

b El coeficiente es significativo al 5\%. 
Tabla 9

Resultados del análisis de regresión múltiple para censos económicos en 2009

\begin{tabular}{|c|c|c|c|c|c|c|c|c|}
\hline \multirow[t]{2}{*}{ Variable dependiente } & \multicolumn{2}{|c|}{ LOG(PROD) } & \multicolumn{2}{|c|}{ LOG(VA) } & \multicolumn{2}{|c|}{ LOG(PROD) } & \multicolumn{2}{|c|}{ LOG(VA) } \\
\hline & Coeficiente & $\mathrm{DE}$ & Coeficiente & $\mathrm{DE}$ & Coeficiente & $\mathrm{DE}$ & Coeficiente & $\mathrm{DE}$ \\
\hline LOG(HORAS)*SEC1 & 0.66 & $0.06^{\mathrm{a}}$ & 0.67 & $0.07^{\mathrm{a}}$ & & & & \\
\hline LOG(FBCF)*SEC1 & 0.19 & $0.04^{\mathrm{a}}$ & 0.23 & $0.05^{\mathrm{a}}$ & & & & \\
\hline LOG(HORAS)*SEC2 & 0.67 & $0.05^{\mathrm{a}}$ & 0.72 & $0.06^{\mathrm{a}}$ & & & & \\
\hline LOG(FBCF)*SEC2 & 0.19 & $0.03^{\mathrm{a}}$ & 0.18 & $0.04^{\mathrm{a}}$ & & & & \\
\hline LOG(HORAS)*SEC3 & 0.001 & 0.11 & 0.28 & $0.11^{\mathrm{b}}$ & & & & \\
\hline LOG(FBCF)*SEC3 & 0.76 & $0.10^{\mathrm{a}}$ & 0.53 & $0.10^{\mathrm{a}}$ & & & & \\
\hline LOG(HORAS)*SEC4 & 0.69 & $0.11^{\mathrm{a}}$ & 0.73 & $0.12^{\mathrm{a}}$ & & & & \\
\hline LOG(FBCF)*SEC4 & 0.25 & $0.11^{\mathrm{b}}$ & 0.22 & $0.10^{\mathrm{b}}$ & & & & \\
\hline $\mathrm{LOG}(\mathrm{PO}) * \mathrm{SEC} 1$ & & & & & 0.62 & $0.08^{\mathrm{a}}$ & 0.68 & $0.08^{\mathrm{a}}$ \\
\hline LOG(ACERVO)*SEC1 & & & & & 0.35 & $0.05^{\mathrm{a}}$ & 0.3 & $0.05^{\mathrm{a}}$ \\
\hline $\mathrm{LOG}(\mathrm{PO}) * \mathrm{SEC} 2$ & & & & & 0.65 & $0.06^{\mathrm{a}}$ & 0.79 & $0.08^{\mathrm{a}}$ \\
\hline LOG(ACERVO)*SEC 2 & & & & & 0.33 & $0.05^{\mathrm{a}}$ & 0.23 & $0.06^{\mathrm{a}}$ \\
\hline $\mathrm{LOG}(\mathrm{PO}) * \mathrm{SEC} 3$ & & & & & -0.01 & 0.2 & 0.3 & 0.2 \\
\hline LOG(ACERVO)*SEC3 & & & & & 0.7 & $0.13^{\mathrm{a}}$ & 0.49 & $0.12^{\mathrm{a}}$ \\
\hline $\mathrm{LOG}(\mathrm{PO}) * \mathrm{SEC} 4$ & & & & & 0.14 & 0.13 & 0.22 & $0.12^{\mathrm{c}}$ \\
\hline LOG(ACERVO)*SEC4 & & & & & 0.67 & $0.08^{\mathrm{a}}$ & 0.59 & $0.08^{\mathrm{a}}$ \\
\hline TAM1 & 5.63 & $0.24^{\mathrm{a}}$ & 4.29 & $0.30^{\mathrm{a}}$ & 3.84 & $0.25^{\mathrm{a}}$ & 3.1 & $0.32^{\mathrm{a}}$ \\
\hline TAM2 & 6.12 & $0.24^{\mathrm{a}}$ & 4.85 & $0.27^{\mathrm{a}}$ & 4.49 & $0.25^{\mathrm{a}}$ & 3.75 & $0.32^{\mathrm{a}}$ \\
\hline TAM3 & 6.56 & $0.29^{\mathrm{a}}$ & 5.29 & $0.32^{\mathrm{a}}$ & 4.87 & $0.28^{\mathrm{a}}$ & 4.17 & $0.36^{\mathrm{a}}$ \\
\hline TAM4 & 6.7 & $0.33^{\mathrm{a}}$ & 5.41 & $0.37^{\mathrm{a}}$ & 4.98 & $0.32^{\mathrm{a}}$ & 4.3 & $0.40^{\mathrm{a}}$ \\
\hline $\mathrm{R} 2$ & & 0.88 & & 0.86 & & 0.91 & & 0.89 \\
\hline R2 ajustado & & 0.88 & & 0.86 & & 0.91 & & 0.89 \\
\hline EE de la regresión & & 0.75 & & 0.81 & & 0.72 & & 0.8 \\
\hline Suma de residuales & & 277 & & 310 & & 290 & & 334 \\
\hline Log likelihood & & -564 & & -579 & & -614 & & -635 \\
\hline Observaciones & & 567 & & 538 & & 567 & & 538 \\
\hline
\end{tabular}

Estimación de la función de producción tipo Cobb-Douglas por sector industrial de base tecnológica y con variables dicotómicas de tamaño de empresa. En las primeras 4 columnas se toman variables explicativas estáticas y en las columnas 5 a 8 se toman variables dinámicas. Se muestran los coeficientes de regresión y los errores estándar. Las regresiones fueron ejecutadas con errores consistentes de White para mitigar problemas de heterocedasticidad.

${ }^{\text {a }}$ El coeficiente es significativo al $1 \%$.
${ }^{b}$ El coeficiente es significativo al $5 \%$.

c El coeficiente es significativo al $10 \%$. 
Tabla 10

Resumen de los rendimientos mostrados en los modelos de regresión 2004 y 2009

\begin{tabular}{|c|c|c|c|c|c|c|c|c|}
\hline \multicolumn{9}{|c|}{ Censos económicos 2004} \\
\hline \multirow[t]{2}{*}{ Variable dependiente } & \multicolumn{2}{|c|}{ LOG(PROD) } & \multicolumn{2}{|l|}{ LOG(VA) } & \multicolumn{2}{|c|}{ LOG(PROD) } & \multicolumn{2}{|l|}{ LOG(VA) } \\
\hline & Coeficiente & $\frac{\text { Rendimiento }}{}$ & Coeficiente & $\frac{\text { Rendimiento }}{}$ & Coeficiente & Rendimiento & Coeficiente & Rendimiento \\
\hline LOG(HORAS)*SEC1 & 0.87 & 1.02 & 0.9 & 1.07 & & & & \\
\hline LOG(FBCF)*SEC1 & 0.15 & & 0.17 & & & & & \\
\hline LOG(HORAS)*SEC2 & 0.85 & 1.01 & 0.91 & 1.05 & & & & \\
\hline LOG(FBCF)*SEC2 & 0.16 & & 0.14 & & & & & \\
\hline LOG(HORAS)*SEC3 & 0.11 & 0.9 & 0.31 & 0.95 & & & & \\
\hline LOG(FBCF)*SEC3 & 0.79 & & 0.64 & & & & & \\
\hline LOG(HORAS)*SEC4 & 0.6 & 1.02 & 0.63 & 1.03 & & & & \\
\hline LOG(FBCF)*SEC4 & 0.42 & & 0.4 & & & & & \\
\hline LOG(PO)*SEC1 & & & & & 0.65 & 1.05 & 0.59 & 1.03 \\
\hline LOG(ACERVO)*SEC1 & & & & & 0.4 & & 0.44 & \\
\hline LOG(PO)*SEC2 & & & & & 0.73 & 1.08 & 0.77 & 1.1 \\
\hline LOG(ACERVO)*SEC2 & & & & & 0.35 & & 0.33 & \\
\hline LOG(PO)*SEC 3 & & & & & 0.15 & 0.83 & 0.81 & 1.09 \\
\hline LOG(ACERVO)*SEC 3 & & & & & 0.68 & & 0.28 & \\
\hline LOG(PO)*SEC4 & & & & & 0.46 & 0.99 & 0.54 & 1.01 \\
\hline LOG(ACERVO)*SEC4 & & & & & 0.53 & & 0.47 & \\
\hline \multicolumn{9}{|c|}{ Censos económicos 2009} \\
\hline LOG(HORAS)*SEC1 & 0.66 & 0.85 & 0.67 & 0.9 & & & & \\
\hline LOG(FBCF)*SEC1 & 0.19 & & 0.23 & & & & & \\
\hline LOG(HORAS)*SEC2 & 0.67 & 0.86 & 0.72 & 0.9 & & & & \\
\hline LOG(FBCF)*SEC2 & 0.19 & & 0.18 & & & & & \\
\hline LOG(HORAS)*SEC3 & 0.001 & 0.761 & 0.28 & 0.81 & & & & \\
\hline LOG(FBCF)*SEC3 & 0.76 & & 0.53 & & & & & \\
\hline LOG(HORAS)*SEC4 & 0.69 & 0.94 & 0.73 & 0.95 & & & & \\
\hline LOG(FBCF)*SEC4 & 0.25 & & 0.22 & & & & & \\
\hline LOG $(\mathrm{PO}) * \mathrm{SEC} 1$ & & & & & 0.62 & 0.97 & 0.68 & 0.98 \\
\hline LOG(ACERVO)*SEC1 & & & & & 0.35 & & 0.3 & \\
\hline $\operatorname{LOG}(\mathrm{PO}) * \mathrm{SEC} 2$ & & & & & 0.65 & 0.98 & 0.79 & 1.02 \\
\hline LOG(ACERVO)*SEC2 & & & & & 0.33 & & 0.23 & \\
\hline $\mathrm{LOG}(\mathrm{PO}) * \mathrm{SEC} 3$ & & & & & -0.01 & 0.69 & 0.3 & 0.79 \\
\hline LOG(ACERVO)*SEC3 & & & & & 0.7 & & 0.49 & \\
\hline LOG(PO)*SEC4 & & & & & 0.14 & 0.81 & 0.22 & 0.81 \\
\hline LOG(ACERVO)*SEC4 & & & & & 0.67 & & 0.59 & \\
\hline
\end{tabular}

En la tabla se muestran los rendimientos de escala para los 4 sectores tecnológicos, tomando en cuenta el trabajo y el capital.

Fuente: Elaboración propia con base en los resultados de las tablas 8 y 9. 
empresas de tamaño grande es mayor que el del resto de los estratos de tamaño. Incluso, se puede decir que el tamaño de la empresa importa, ya que a mayor tamaño de la empresa se tiene un nivel tecnológico mayor.

En contraste con los resultados mostrados en la tabla 9, se puede observar que en la mayoría de los casos no existen rendimientos de escala crecientes ni constantes debido a que en ninguna de las regresiones se estiman estas sumas en los coeficientes de $\mathrm{K}$ y L.

\section{Resultados del análisis de regresión múltiple para censos económicos de 2009}

Por otra parte, en los censos de 2009 también importa el tamaño de la empresa, ya que a mayor tamaño de la empresa se parte de un nivel tecnológico mayor, lo cual implica una mayor capacidad de producción.

Se muestra, de esta manera, que aunque se tenían en su mayor parte economías de escala con rendimientos crecientes para los censos de 2004, para los censos de 2009 esto no se sostiene. En las tablas 4 y 5, mostradas previamente, se puede inferir que aunque no se tienen economías de escala con rendimientos crecientes en 2009, el nivel de participación de la producción de las EBT en la economía nacional crece en un 2\%, pasando del 20 al 22\%, y manteniendo un nivel de participación de generación de valor agregado de un 13\%. Esto demuestra que las EBT no requieren de rendimientos crecientes para lograr el éxito empresarial. Esta evidencia es muy significativa para probar la pertinencia de la segunda hipótesis de investigación.

En las tablas 8 y 9, presentadas previamente, se puede observar que el nivel tecnológico de las EBT está directamente relacionado con el tamaño de la empresa (ver los coeficientes de variable TAM1, TAM2, TAM3 y TAM4 en las tablas 8 y 9). Estos resultados permiten plantear que una EBT de tamaño pequeño tiene mayores niveles de tecnología que las EBT de tamaño más pequeño, confirmando la tercera hipótesis de la investigación. Estos resultados permiten afirmar que la MIPYME genera un mayor nivel de valor agregado, y que, por otra parte, este valor agregado no depende de la escala de producción con rendimientos crecientes.

En la tabla 10 se muestra un resumen de ambos modelos de regresión, en donde se evidencia que en prácticamente ninguno de los sectores para el año 2009 se tienen rendimientos crecientes de escala; de hecho, los rendimientos son decrecientes, en tanto que para el año 2004 los rendimientos fueron crecientes para prácticamente todos los sectores tecnológicos.

\section{Conclusiones}

Se ha encontrado como principal hallazgo que las microempresas de este tipo generan mayores volúmenes de valor agregado que las empresas de tamaño más grande. Por otra parte, se corroboró que no es la escala de producción con rendimientos crecientes la que determina el éxito de este tipo de empresas.

Se identificó que para los censos de 2004 y 2009 se tienen rendimientos crecientes y rendimientos decrecientes, y a pesar de esta condición, se mantienen los montos de producción y empleo de las EBT. Por último, se confirma que a pesar de no ser las grandes EBT las que generan mayores volúmenes relativos de valor agregado, sí son estos estratos de empresa los que manejan mayores niveles tecnológicos.

Otros resultados interesantes residen en la contribución a la producción de estos sectores tecnológicos. Para ambos periodos se tiene que aportan más del $20 \%$ del total de la producción, y una generación del 13\% del valor agregado nacional, con solo el $8.2 \%$ del total del personal ocupado a nivel nacional. Este dato pone de manifiesto la rentabilidad de estos sectores. Un último 
resultado interesante es el hecho de que la mayor parte de estas empresas son de tamaño micro. Esto sucede con la estructura empresarial en general, con la diferencia de que estas empresas con relativamente poco tiempo de vida y con pocas personas ocupadas generan montos de producción y valor agregado significativos a nivel nacional.

Algunas limitaciones de este estudio atienden al tiempo en que se tomaron los datos, pues la información más actualizada de estos sectores está en los censos económicos. El censo más actual es el censo de 2009, por lo que aún nos queda un año para tener acceso a los nuevos censos de 2014. Adicionalmente, en el modelo de regresión se toman datos solo de factores como el capital y el trabajo, dejando de lado, por simplicidad en el modelo y disponibilidad de datos, otros factores. Algunos de estos factores son: el nivel de capital de las tecnologías de la información, las materias primas, el nivel de capacitación y algunas otras variables factoriales, así como variables de control. Sin embargo, los resultados son significativos e ilustrativos del funcionamiento del sector.

Una limitación final tiene que ver con el nivel de desglose de las ramas tomadas en cuenta para construir los sectores tecnológicos. En algunas ramas aún se podría hacer una medición más precisa si los datos lo permiten. Un ejemplo es la rama 6215, «Laboratorios médicos y de diagnóstico», en la cual existen laboratorios de diagnóstico a pacientes y laboratorios de construcción de equipo médico. El primero constituye un sector con baja intensidad tecnológica, mientras que el segundo constituye un sector de tecnología de punta.

\section{Referencias}

Aspelund, A., Berg, T. y Skjevdal, R. (2005). Initial resources' influence on new venture survival: A longitudinal study of new technology-based firms. Technovation, 25, 1337-1347.

Autio, E. y Yli-Renko, H. (1998). New, technology-based firms in small open economies-An analysis based on the Finnish experience. Research Policy, 26, 973-987.

Barney, J. B. (1986). Strategic factor markets: Expectations, luck, and business strategy. Management Science, 32 , 1231-1241.

Bollinger, L., Hope, K. y Utterback, J. (1983). A review of literature and hypothesis on new technology based firms. Research Policy, 12, 1-14.

Breschi, S., Lenzi, C., Malerba, F. y Mancusi, M. (2014). Knowledge-intensive entrepreneurship: Sectoral patterns in a sample of European high-tech firms. Technology Analysis \& Strategic Management, 26, 1-14.

Butchart, R. (1987). A new UK definition of high technology industries. Economic Review, 400, 82-88.

Clarysse, B., Bruneel, J. y Wright, M. (2011). Explaining growth paths of young technology-based firms: Structuring resource portfolios in different competitive environments. Strategic Entrepreneurship Journal, 5, 137-157.

Coase, R. (1937). The nature of the firm. Economica London School of Economics, 4, 386-405.

Coeurderoy, R. y Murray, G. (2008). Regulatory environments and the location decision: Evidence from the early foreign market entries of new-technology-based firms. Journal of International Business Studies, 39, 670-687.

Colombo, M. y Grilli, L. (2005). Founders' human capital and the growth of new technology-based firms: A competencebased view. Research Policy, 34, 795-816.

Dierickx, I. y Cool, K. (1989). Asset stock accumulation and sustainability of competitive advantage. Management Science, $35,1504-1513$

Ejermo, O. y Xiao, J. (2014). Entrepreneurship and survival over the business cycle: How do new technology-based firms differ? Small Business Economics, 1, 1-16.

Fong, C. R. y Alarcón, M. A. (2010). Funcionamiento de empresas de base tecnológica: obtención de recursos estratégicos en las junturas críticas. Revista Internacional de Administración y Finanzas, 3, 39-54.

Fontes, M. y Coombs, R. (2001). Contribution of new technology-based firms to the strengthening of technological capabilities in intermediate economies. Research Policy, 30, 79-97.

Ganotakis, P. y Love, J. (2011). R\&D, product innovation, and exporting: Evidence from UK new technology based firms. Oxford Economic Papers, 63, 279-306.

Granstrand, O. (1998). Towards a theory of the technology-based firm. Research Policy, 27, 465-489. 
Grant, R. M. (1991). The resource-based theory of competitive advantage: Implications for strategy formulation. California Management Review, 33, 114-135.

Instituto Nacional de Estadística y Geografía. Censos Económicos de 2009 [consultado Ene 2014]. Disponible en: http://www.inegi.org.mx/est/contenidos/Proyectos/ce/default.aspx

Kollmer, H. y Dowling, M. (2004). Licensing as a commercialisation strategy for new technology-based firms. Reseach Policy, 33, 1141-1151.

Kulicke, M. y Krupp, H. (1987). The formation, relevance and public promotion of new technology-based firms. Technovation, 6, 47-56.

Lall, S. (2000). The technological structure and performance of developing country manufactured exports, 1985-98. Oxford Development Studies, 28, 337-369.

Li, L., Qian, G. y Qian, Z. (2012). The performance of small and medium-sized technology-based enterprises: Do product diversity and international diversity matter? International Business Review, 21, 941-956.

Lockett, A. y Wright, M. (2005). Resources, capabilities, risk capital and the creation of university spin-out companies. Research Policy, 34, 1043-1057.

March, I. y Yagüe, R. (1999). A new tool to classifying new technology-based firm prospect and expectations. The Journal of High Technology Management Research, 10, 347-376.

Oakey, R, Groen A., van der Sijde, P., Cook, G. (1993). New technology-based firms in the new millennium. Emerald Book Series, Vol. 1.

Onetti, A., Zucchella, A., Jones, M. y McDougall, P. (2012). Internationalization, innovation and entrepreneurship: Business models for new technology-based firms. Journal of Management \& Governance, 16, 337-368.

Ortin, P. y Vendrell, F. (2014). University spin-offs vs. other NTBFs: Total factor productivity differences at outset and evolution. Technovation, 34, 101-112.

Patel, P. y Pavitt, K. (1994). Technological competencies in the world's largest firms: Characteristics, constraints and scope for managerial choice. STEEP Discussion Paper No. 13, Science Policy Research Unit, University of Sussex.

Pavitt, K., Robson, M. y Townsend, J. (1989). Technological accumulation, diversification and organisation in UK companies, 1945-1983. Management Science, 35.

Rae, D. (2006). Entrepreneurial learning: A conceptual framework for technology-based enterprise. Technology Analysis \& Strategic Management, 18, 39-56.

Solow, R. M. (1957). Technical change and the aggregate production function. The Review of Economics and Statistics, $39,312-320$.

Storey, D. J. y Tether, B. S. (1998a). New technology-based firms in the European Union: An introduction. Research Policy, 26, 933-946.

Storey, D. J. y Tether, B. S. (1998b). Public policy measures to support new technology-based firms in the European Union. Research Policy, 26, 1037-1057.

Suzuki de Oliveira, R., Teixeira de Freitas, A., Ferreira, M. y Real, N. (2011). Analysis of competences for innovation in technology-based enterprise incubators. Latin American Business Review, 12, 187-207.

Teixeira, A. y Tavares, T. (2014). Human capital intensity in technology-based firms located in Portugal: Does foreign ownership matter? Research Policy, 43, 737-748.

Tsvetkova, A., Thill, J. y Strumsky, D. (2014). Metropolitan innovation, firm size, and business survival in a high-tech industry. Small Business Economics, 43, 661-676.

Wernerfelt, B. (1984). A resource-based view of the firm. Strategic Management Journal, 5, 171-180.

Wu, L. Y. y Wang, C. J. (2007). Transforming resources to improve performance of technology-based firms: A Taiwanese Empirical Study. Journal of Engineering and Technology Management, 24, 251-261.

Yagüe, R. M. y Mach, I. C. (2013). Performance analysis of NTBFs in knowledge-intensive industries: Evidence from the human health sector. Journal of Business Research, 66, 1983-1989. 